\title{
Quasicrystal Structures
}

\section{Progress in Understanding}

\author{
C. Janot \\ Institut Laue-Langevin, Grenoble, France
}

Quasicrystals first achieved prominence in 1984 following the report by Schechtman, Blech, Gratias and Cahn of metallic alloys with very surprising diffraction properties. The new alloys, notably rapidly solidified $\mathrm{Al}-\mathrm{Mn}$ compounds, produced electron diffraction patterns consisting of sharp spots but showing five-fold symmetries. The patterns were surprising because sharp diffraction peaks are normally associated with periodic crystal structures, yet the sacred rules of crystallography forbid five-fold symmetry. Since then, numerous other metallic alloys displaying various "forbidden" symmetries have been discovered. The challenge has been to find models for the atomic structures, and to investigate them experimentally.

Neutron diffraction patterns, showing contrast variation effects have, in particular, provided reasonable insights so that it is possible to say where most of the atoms are located. It is also now accepted that "perfect" quasicrystals do exist, which means that quasiperiodicity can be obtained without intrinsic disorder. This clearly opens the way to the possible discovery of properties that would be characteristic of quasicrystals.

Long-range ordered structures do not necessarily result from periodic space tiling and the concept of a quasicrystal, or a quasiperiodic crystal, has been introduced to describe substances that have a sharply peaked diffraction pattern without any evidence for periodicity. More precisely, quasiperiodic functions are defined mathematically as irrational three-dimensional cuts through objects which are periodically distributed in a higher-dimensional space. It is this approach that will be described and illustrated.

Professor Christian Janot has been on secondment to the Institute Laue-Langevin, avenue des Martyrs / 156 X, 38042 Grenoble Cedex, France from the Physics Department, University of Nancy since 1982. He is a physical metallurgist interested in the structures of disordered systems.

\section{Quasicrystallography}

For periodic crystals, the structure is completely specified when both the unit cell (or the Bravais lattice) and the positions of atoms in this unit cell have been determined. The so-called direct methods of crystallography are usually used to extract this structural information from diffraction data.

Quasicrystals must be treated in essentially the same way: positioning (indexing) of the diffraction peaks is related to the geometrical characteristics of the quasi-periodic framework, while the distribution of scattered intensity should reveal where the atoms are located. The first step, i.e. description of the quasiperiodic lattice, has been achieved using a variety of different schemes for generating the lattices. In particular, the so-called cut-projection method is nothing more than an explicit statement of the definition of quasiperiodicity whereby any 3-d quasiperiodic lattice has hidden translation invariances which can be recovered if the structure is properly described in a higher-dimensional space. For instance, archetypical icosahedral quasicrystals with $m \overline{3} \overline{5}$ point-group symmetries cannot be periodic in 3-d. However, there are three possible 6-d, icosahedral periodic arrangements, i.e. the primitive $(P)$, facecentred ( $F$ ) and body-centred (I) cubic Bravais lattices. Each of these yields well defined indexing (positions and extinction rules) of diffraction peaks.

The second step, i.e. saying where the atoms are, is intrinsically more difficult for a quasicrystal than for a crystal. A perfect quasi-periodic structure without any disorder still has an infinite number of sites in three dimensions which are not exactly equivalent. There are also practical difficulties owing to the fairly low level of information that can be extracted from diffraction patterns.

\section{The cut-projection method}

To illustrate the relationship between diffraction data and the structure of quasicrystals for the cut-projection method, a simple low-dimensional example is shown in Fig. 1. Here a 1-d quasipe-

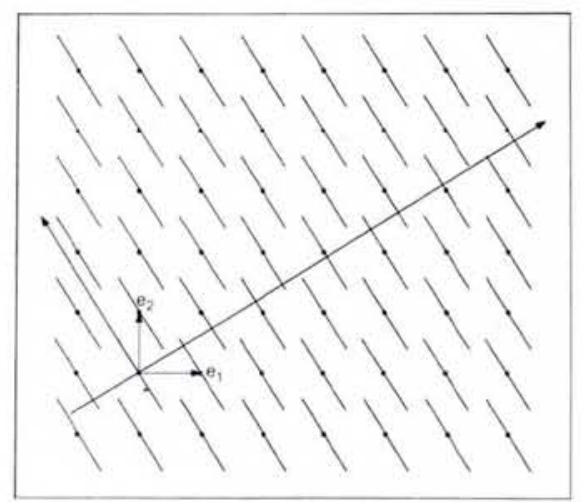

Fig. 1 - Illustration of the cut-projection method for the case of a periodic 2-d aperiodic 1-d relation. The basis associated with the 2-d square lattice is a single line segment perpendicular to the real 1-d space. The intersection is a quasicrystal formed by the stacking of two different tiles, with lengths $L$ and $S$, in a Fibonacci sequence.

riodic chain corresponds to a periodic square lattice arrangement of segments (called $A_{\text {perp }}$ ). The physical space containing the quasiperiodic chain is called parallel (par) space while the complementary space (containing $A_{\text {perp }}$ in this example) is the perpendicular (perp) space. The $A_{\text {perp }}$ segments intersect the 1-d real world space at the atom positions (generally speaking, the $A_{\text {perp }}$ are not necessarily "flat", they cannot intersect each other, and they have to be invariant under the operations of the point group symmetry).

In Fig. 1, the slope of the physical parallel space into the 2-d hyperspace has been chosen to generate a so-called Fibonacci chain: two tiles, Large and Short, arranged in a sequence $L S L S L L$ SLLSLSL..., with $L / S=\tau$ the golden mean. The deterministic construction rules of a Fibonacci chain are such that starting with $L S$, one goes on and on replacing infinitely $L$ by $L S$ and $S$ by $L$.

A calculated diffraction pattern for the Fibonacci chain is shown in Fig. 2. It is a singularly continuous set of Bragg peaks, most of which are, for practical reasons, smeared out into background 


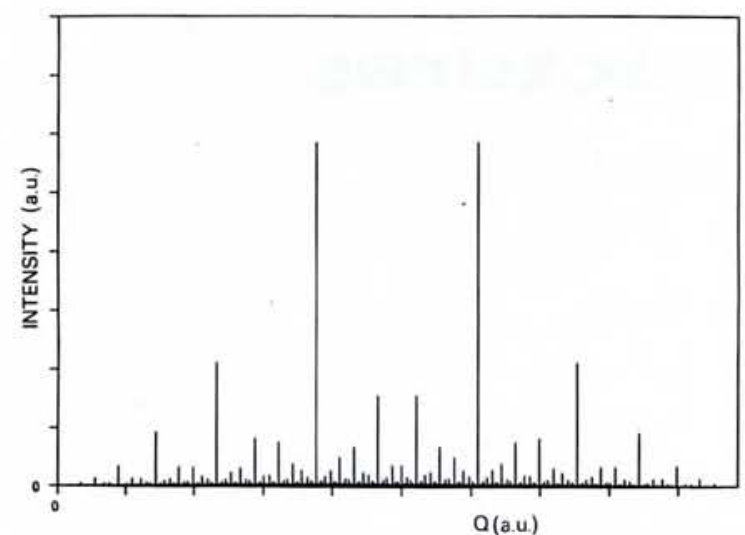

Fig. 2 - Fourier spectrum of a Fibonacci sequence calculated using 987 integration steps.

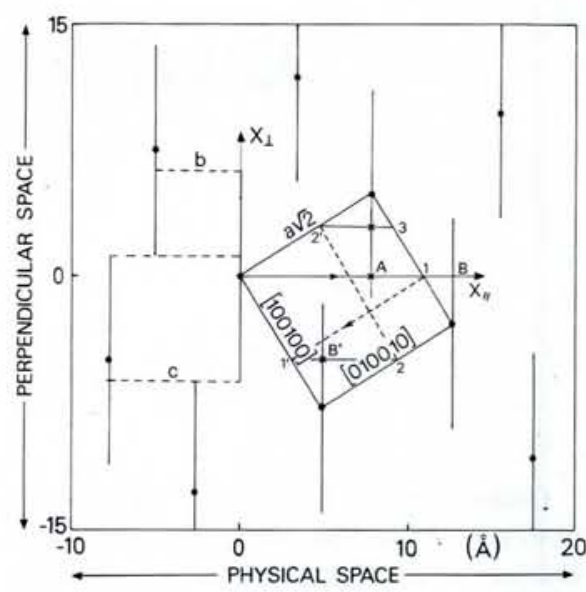

Fig. 3 - A two-dimensional slice of 6-d primitive cubic lattice with a triacontahedral hypersurface $A 3_{\text {perp }}$ attached to each node. The cut contains a pair of two-fold axes $\left(x_{\text {par }}\right.$ in the physical space and $x_{\text {perp }}$ in the complementary - or perpendicular space), nodes of the 6-d lattice (dots in the figure) and a cut of $A 3_{\text {perp }}$ (segments in the figure). Squares delimited by dashed lines show the occurrence of atomic spacings $b$ and $c$ along the face diagonals of the 3-d Penrose tiles. $A$ and $B$ (or equivalently $B^{\prime}$ ) are examples of atom positions located on two-fold axes in the physical 3-d space.

site positions and $A 3$ volumes (size and shape), the later requiring an infinite number of parameters to be specified. Modelling therefore cannot be completely avoided.

\section{Isotope substitution}

In contrast to the examples described so far, real quasicrystals are neither monatomic nor single-sited systems. Consequently, only combinations of the different convoluted substructures can emerge from trivial diffraction approa- ches. This difficulty has been overcome using contrast variation effects. A good example of contrast variation is found in isotope substitution coupled with neutron diffraction. Different isotopes of a chemical element scatter neutrons differently, sometimes with changes in both amplitudes and phases $\left(e . g^{6} \mathrm{Li}\right.$ and ${ }^{7} \mathrm{Li},{ }^{62} \mathrm{Ni}$ and ${ }^{58} \mathrm{Ni}$, etc.). By measuring neutron diffraction patterns of samspace only. Thus, only the few Bragg peaks that are not too far removed in the reciprocal complementary space project as strong features onto the parallel reciprocal space, while a large number of them are not measurable. This explains the singularly continuous aspect of the 1-d diffraction pattern as being due to the irrationality of the projection effect. Hence the Fourier transform of a correctly indexed diffraction pattern in principle describes the periodic hyperspace structure.

The 3-d analog of a Fibonacci chain is a 3-d Penrose tiling (3DPT) which can be constructed by assembling two different, properly tailored rhombohedral tiles. The related hyperspace now has to be six dimensional, with a 6-d primitive cubic lattice and $A_{\text {perp }}$ segments replaced by triacontahedral $A 3_{\text {perp }}$ atomic volumes. Our 3-d physical space must be embedded into six dimensions in such a way that the six eigen directions of the structure project along the six five-fold axes of an icosahedron. The 6-d structure, and the 3-d cut procedure

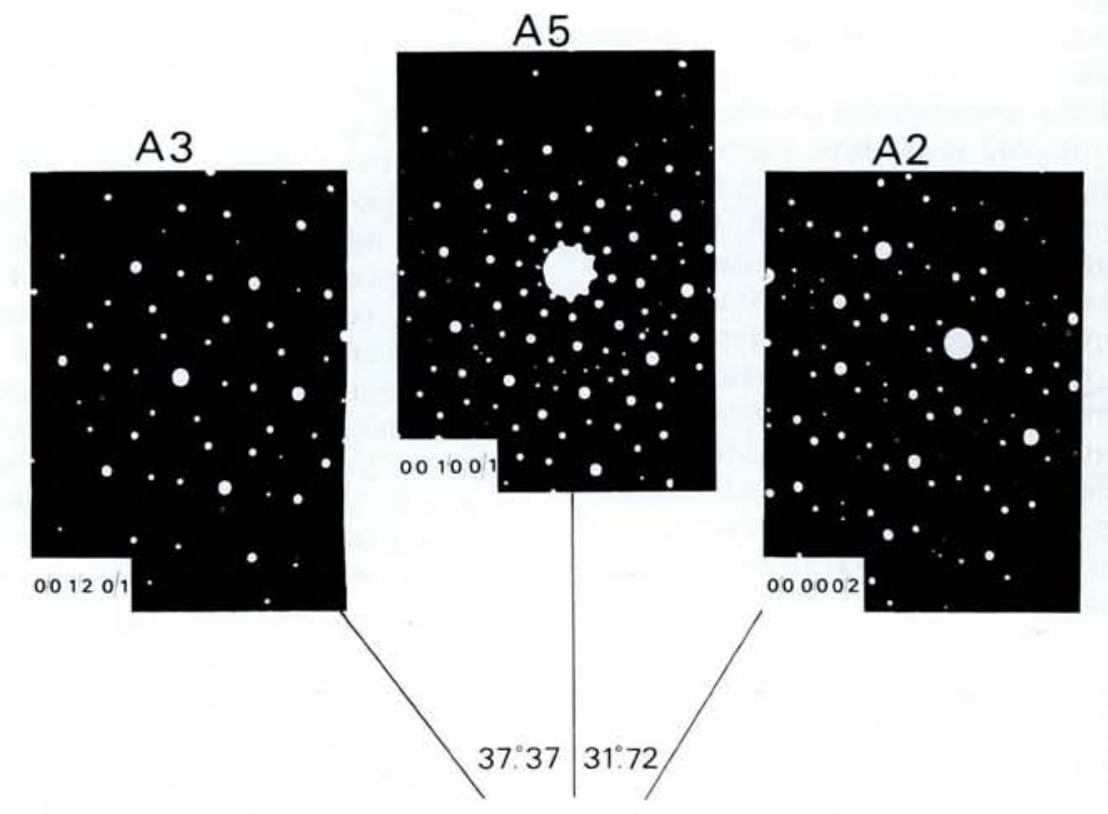

Fig. 4 - Electron diffraction patterns showing different zone axes for a AIMn quasicrystal (indicated indexing is with respect to the 6-d periodic structure). 
ples containing different isotopic compositions it is possible to extract "partial" structure factors corresponding to each "one-atom" substructure. Isomorphous substitution is also to some extent achievable.

\section{Where are the Atoms Located?}

Although thirty or so different quasicrystals have been discovered so far, detailed structural investigations have been mainly restricted to a few icosahedral phases. The first indication of an icosahedral phase usually comes from electron diffraction and electron microscopy. By rotating the specimen, successive diffraction patterns can be obtained which are typical for five-fold (6 directions), three-fold (10 directions) and two-fold (15 directions) zone axes (Fig. 4). Convergent beam electron diffraction and contrast variation effects in neutron diffraction can then establish the existence of an inversion centre. The icosahedral point group $m \overline{3} \overline{5}$ has thus been assigned to the structures of the AIMn, AlLiCu and AlFeCu quasicrystalline phases.

\section{AlLiCu quasicrystals}

Structural investigations of AlLiCu quasicrystals by $\mathrm{X}$-ray or neutron diffraction were hampered for a while by the lack of large (quasi)single crystals. These investigations have taken a leap forward with the availability of grains approaching a millimetre across (Fig. 5). Peaks observed in four-circle single

Fig. 5 - Triacontahedral single grain of the AlLiCu icosahedral phase (courtesy of $P$. Sainfort, Pechiney Research Centre).

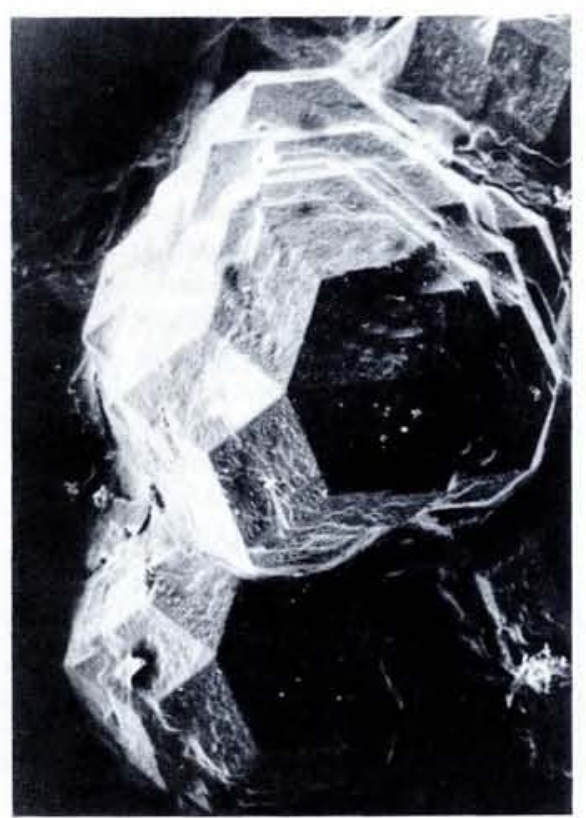

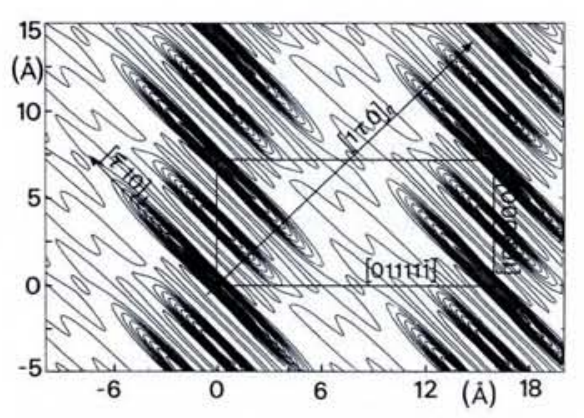

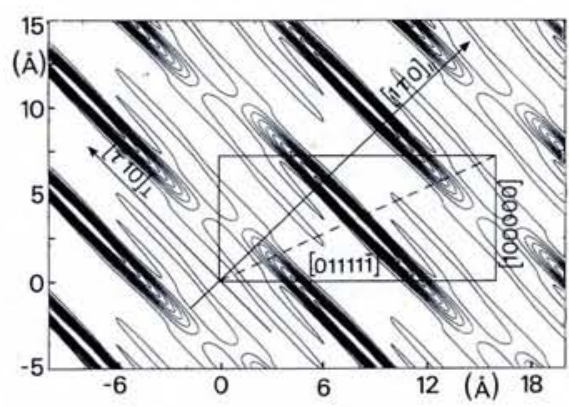

Fig. 6 - Examples of 2-d slices of the experimentally determined $6-d$ density for an AlLiCu quasicrystal for the $A(A l+C u)-$ upper - and the Li sublattice-lower. The slices are taken along five-fold axes, one in physical space, the other in complementary space. Atom positions are given by the intersection of the physical five-fold axis with the density features.

crystal diffraction scans have been found to be mostly indexable with six indices that belong to a primitive icosahedral Bravais lattice having a lattice parameter of $7.15 \AA$. The true composition of the icosahedral phase is $\mathrm{Al}_{5.70} \mathrm{Cu}_{1.08} \mathrm{Li}_{3.22}$, which is not too far from that of the so-called crystalline $R$-phase $\mathrm{Al}_{5.60} \mathrm{Cu}_{1.20} \mathrm{Li}_{3.20}$, so allowing comparisons of the structures.

It has been demonstrated that $\mathrm{Al}+\mathrm{Cu}$ atoms ( $A$ atoms hereafter) occupy a family of positions with little, if any, order while $A$ and $\mathrm{Li}$ atoms are chemically ordered. This pseudo-binary alloy behaviour has greatly simplified the structure specification. Partial structure factors, with both amplitudes and phases, have been determined for the two $A$ and $\mathrm{Li}$ substructures. Fourier transforms of these partial structure factors have resulted in the 6-d density distributions, as illustrated by the 2-d slices of the 6-d space shown in Fig. 6. Clearly, description of the quasicrystal density is simplified if it is analysed in terms of a 2-d periodic density.

The density corresponding to $A$ atoms $(\mathrm{Al}+\mathrm{Cu})$ is mostly concentrated in two types of narrow, almost 3-d regions, perpendicular to the physical space and centred at the vertices and midedge positions of the hypercubic lattice (Fig. 6a). Projections onto the 3-d perpendicular space, shown in Fig. 7a, reveal detailed shape features of these $A 3_{\text {perp }}$ volumes. Similarly, the lithium density shows up in bodycentre regions (Figs. 6b and 7b).

Atom positions in the 3-d physical space are obtained by noting intersections of the 6-d density distribution with any direction in the physical 3-d space. But how can the 3-d structure be described without generating a tedious list of atom coordinates? There are two complementary descriptions of the Al$\mathrm{LiCu}$ atomic arrangement. One is an average decoration of the two tiles of a 3DPT, with sites for $A$ atoms $(\mathrm{Al}+\mathrm{Cu})$ at the vertices and mid-edges, and sites for $\mathrm{Li}$ atoms on the long diagonals of the big rhombohedra (Fig. 8). Decoration is non-uniform and varies from tile to tile, implying that "average" sites are only partially occupied. There is no true 3DPT and such a description, although pictorial, may be misleading.

The alternative and more fruitful description of the structure is related to the parent crystalline $R$-phase. Similar atomic shells are found in both phases, with differences coming from size changes, more perfect icosahedrality in the quasicrystal and a different bonding scheme for larger clusters.
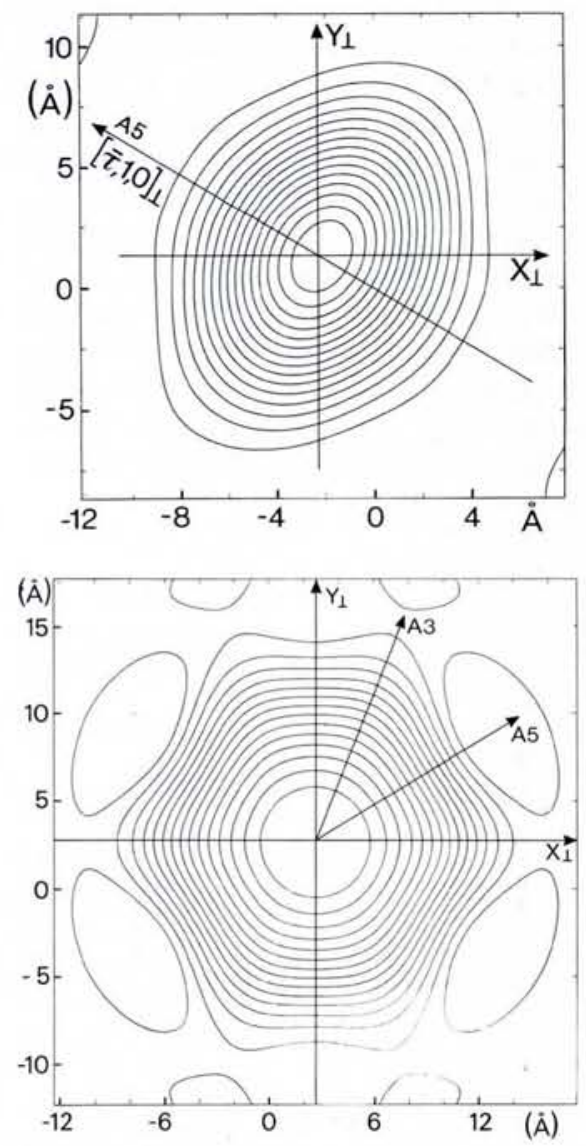

Fig. 7 - Projections onto the 3-d perpendicular space of the $A 3_{\text {perp }}$ volumes corresponding to the density features shown in Fig. 6 ( $A$ atoms-upper; Li atoms-lower). 


\section{AlSiMn quasicrystals}

Historically, the $\mathrm{Al}_{74} \mathrm{Si}_{5} \mathrm{Mn}_{21}$ quasicrystal came before AlLiCu, but a complete specification of its structure is still underway. The average 3-d decoration which has been proposed is related to a more complicated 6-d periodic density owing to $A 3_{\text {perp }}$ volumes not really being $3-d$ but showing a significant physical space component. The 6-d lattice for $\mathrm{Al}_{74} \mathrm{Si}_{5} \mathrm{Mn}_{21}$ is, as for $\mathrm{Al}_{5.70} \mathrm{Cu}_{1.08} \mathrm{Li}_{3.22}$, a primitive Bravais lattice but with different density distributions. Diffraction studies are less accurate because single (quasi)crystals are lacking.

\section{The Future}

Alternative descriptions based on giant cell periodic crystals, twinned crystals or glasses of icosahedral clusters have been proposed for quasicrystals. Distinguishing between these models is not an easy matter, although electron diffraction and high resolution electron microscopy images have provided support for the fully ordered, quasiperiodic lattice model.

A recently discovered quasicrystal in the AlFeCu system may help to settle the issue once and for all. Its many interesting features have already prompted a wide variety of structural and physical studies. The AIFeCu quasicrystalline phase apparently forms as a stable phase via a peritectic reaction from a crystalline primary phase. High quality single grains with dodecahedral faceting can be grown such that the widths of diffraction peaks are limited only by the experimental resolution. Preliminary structural studies show that a chemical order-disorder transition exists where the ordered phase corresponds to a face centered 6-d hypercubic lattice.
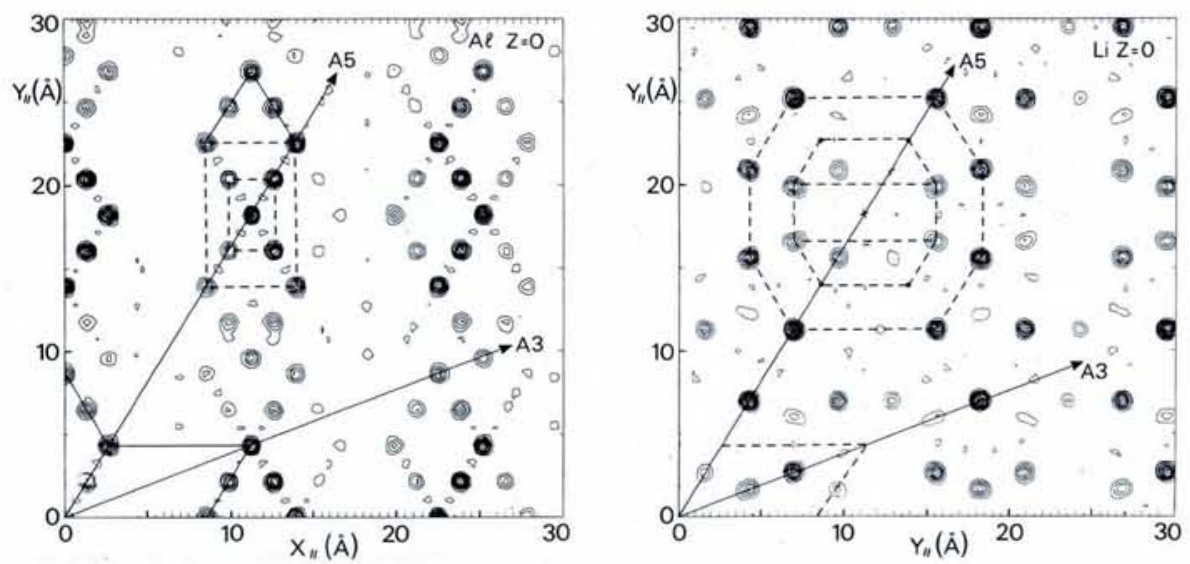

Fig. 8 - Examples of 2-d slices of the physical 3-d density for the AlLiCu quasicrystal as deduced from a cut of the 6-d experimental structure. Midedge and vertex decoration of a 3DPT Penrose tiling are shown for $A$ atoms (left); diagonal sites appear for Li atoms (right).

\section{Edoardo Amaldi}

The European physics community, together with all its Italian colleagues and friends, was deeply sorrowed by the sudden death of Edoardo Amaldi on the fifth of December last year in Rome while going to his office at the Accademia dei Lincei, where he was the President.

Amaldi was among the eminent physicists who promoted the foundation of the EPS in Pisa 22 years ago. He continued to maintain a keen interest in the Society, and as an honorary member played a vital role in encouraging collaboration at a truly European level. His work toward furthering cooperation and mutual understanding between eastern and western European countries has a symbolic relevance to-day. Nor should we forget the important and useful advice he offered during his uninterrupted participation over many years in the activities of the Italian Physical Society.

It may even be superfluous to mention his contribution to the creation and development of CERN, acting as Secretary-General of the Council which gave rise to the organization's acronym. He later became the first President of the European Committee for Future Accelerators (ECFA), and in 1963 presented the report that mapped the future of high energy physics in Europe.

In Italy, as professor of physics at the University of Rome since 1937, Edoardo Amaldi was always considered a true Master, leading a large number of physicists in different fields of research and teaching. He figured prominently in the revival of physics in Italy after the Second World War along the lines chartered by Fermi and Majorana. The foundation of the Instituto Nazionale di Fisica Nucleare (INFN) in 1951 - Amaldi was its second President after Bernardini - exemplifies the creation of a very efficient institution which has contributed greatly to the international prestige of Italian science.

The outstanding scientific career of Edoardo Amaldi stretches from his legendary work in nuclear physics with Fermi at the "Via Panisperna" Institute in Rome in the 1930's, to his involvement at the time of his death in research on gravitational waves. One cannot give proper credit in this short summary to his keen interest in the applied and social aspects of science, including the peaceful use of nuclear energy and nuclear disarmament, to which he contributed greatly.

To remember Edoardo Amaldi correctly we need his remarkable ability to recreate the scientific and personal life of an historical figure, as he himself demonstrated for Majorana and Touscheck. His wonderful personality bore witness to the importance of carrying out true research without any concessions; it is a shining example for young physicists.

R.A. Ricci, INFN, Padova 